\title{
Procesos de conversión de acero y efecto de variables en convertidores tipo L.B.E..$^{(\bullet)}$
}

\author{
C. $\operatorname{Blanco}^{(*)}$, A. Vega $^{(*)}$ y M. Díaz ${ }^{(*)}$ \\ Resumen Se analiza la eficacia de los procesos de transformación en convertidores en condiciones de trabajo \\ determinadas, para deducir relaciones aplicables al control basadas en la modificación de cantidades \\ y composiciones de las materias primas. Se plantean ecuaciones empíricas para determinar la compo- \\ sición final del acero como función de los aditivos y de las concentraciones iniciales del arrabio tra- \\ tado, así como el patrón de soplado de oxígeno. Las relaciones obtenidas establecen las dependencias \\ entre cada una de las variables consideradas y, por tanto, permiten determinar estrategias de opera- \\ ción para obtener aceros con unas calidades finales dadas. \\ Palabras clave: Acero. Convertidor. Modelo estadístico. Control.

\section{Steel conversion processes and effect of the variables in L.B.E. converters}

\begin{abstract}
In order to determine control equations based on qualitative and quantitative changes of the raw materials, an analysis of the efficiency of the steel production process was carried out in converters under specified operation conditions. Empirical equations to obtain the final composition of the steel as a function of the additives, the initial concentrations of the hot metal and the blowing oxygen patterns are established. The resulting relationships depend on the considered variables and therefore could be used to determine operational strategies in order to obtain steels of a certain quality.
\end{abstract}

Keywords: Steel. Converter. Statistical model. Control.

\section{INTRODUCCIÓN}

El comportamiento de un convertidor de acero resulta complejo por el gran número de variables que lo caracterizan, fases de reacción implicadas y reacciones que tienen lugar. La aplicación de métodos basados en relacionar estas variables con las condiciones iniciales de trabajo ya han sido utilizadas en otro tipo de operaciones. Así, en hornos altos (1), cuyo comportamiento resulta igualmente complejo debido al gran número de variables que caracterizan el sistema, el efecto de cada una de éstas se ha estudiado a través de un análisis de los datos de planta, estableciéndose ecuaciones de regresión lineal y multilineal que expresan la cantidad de carbono al final de la combustión y la productividad

\footnotetext{
(•) Trabajo recibido el día 30 de enero de 1995.

(*) Dpto. de Ingeniería Química. Universidad de Oviedo. 33071-Oviedo (España)
}

como función de las variables independientes que tienen mayor influencia.

En este trabajo se analizan los datos de planta procedentes de un convertidor de acero de ENSIDESA para establecer relaciones empíricas y, por tanto, determinar la dependencia entre estas variables. Las variables estudiadas son: 1) composición del acero, es decir, porcentajes finales de carbono, manganeso, silicio, fósforo, azufre, y 2) composición de la escoria: $\mathrm{SiO}_{2}, \mathrm{MnO}, \mathrm{P}_{2} \mathrm{O}_{5}, \mathrm{~S}, \mathrm{TiO}_{2}, \mathrm{Fe}_{\mathrm{t}} \mathrm{O}$, $\mathrm{CaO}, \mathrm{MgO}, \mathrm{Al}_{2} \mathrm{O}_{3}$. Todas estas variables se han estudiado en función de las iniciales de entrada: aditivos añadidos, oxígeno soplado, composición y temperatura iniciales del arrabio utilizado. A partir de los ajustes realizados y analizando las dependencias entre las distintas variables, se pueden obtener estrategias de operación para el oxígeno soplado, aditivos añadidos $\mathrm{y}$, por tanto, determinar para cada caso cuál debe ser la estrategia de operación más adecuada para obtener el producto final dentro de los límites de calidad impuestos. 


\section{SELECCIÓN DE LAS VARIABLES UTILIZADAS}

Es importante tener en cuenta que el uso de las relaciones obtenidas sólo podrán aplicarse cuando las condiciones de trabajo sean las mismas que las que se han utilizado para realizar los ajustes estadísticos. Por ello, siempre que se alteren las condiciones de operación, es necesario obtener nuevas ecuaciones empíricas. Las ecuaciones resultantes de los análisis estadísticos pueden aplicarse a la operación y al control de convertidores y, en particular, a la optimización de las cargas iniciales. El conocimiento de los elementos más interrelacionados a través del análisis de la varianza permite disponer de criterios de decisión de los elementos sobre los que actuar cuando se dispone de métodos de control.

El afino del acero en los convertidores tiene como principal objetivo obtener un acero con unas características definidas en composición y temperatura adecuadas, para un posterior tratamiento donde se consiguen las especificaciones requeridas en la elaboración del acero (2).

El proceso parte de un arrabio inicial procedente de hornos altos con una composición y una temperatura determinadas. Las variables que se van a tener en cuenta son las que se recogen en la tabla I.

En el estudio estadístico inicial se analizaron las correlaciones entre las variables, encontrando que entre algunas de ellas existe una correlación importante, lo que hace difícil la explicación de los efectos que producen determinadas variables sobre la variable dependiente; sin embargo, en este estudio, sólo se admitieron aquellos ajustes que permiten explicar adecuadamente los efectos fisicoquímicos del proceso, ya que determinar cómo afectan a la variable dependiente variables correlacionadas resulta muy complejo y, actualmente, no se encuentran formas de esclarecer el efecto de variables correlacionadas totalmente satisfactorias.

\subsection{Variables independientes}

\subsubsection{Composición del arrabio}

- Concentración de carbono, manganeso, silicio, fósforo y azufre; temperatura inicial y peso del arrabio añadido.

\subsubsection{Variables de operación}

- Patrón de soplado: oxígeno total soplado, así como la distribución de éste por tiempos parciales a lo largo del proceso.

- Aditivos: espato, precoque, cal, coque, dolomía cruda, dolomía calcinada, sínter y chatarra de hierro.
TABLA I.- Variables consideradas en los ajustes estadísticos realizados

TABLE I.- Considered variables in the statistical analysis

Variables iniciales: Variables independientes

\begin{tabular}{|c|c|l|}
\hline \multirow{2}{*}{$\begin{array}{c}\text { Composición } \\
\text { del } \\
\text { arrabio }\end{array}$} & \multicolumn{2}{|c|}{ Variables de operación } \\
\cline { 2 - 3 } & $\begin{array}{c}\text { Patrón de } \\
\text { soplado }\end{array}$ & Adicionantes \\
\hline $\mathrm{C}_{0}$ & ign & Espato \\
$\mathrm{Mn}_{0}$ & $\mathrm{IM}$ & Precoque \\
$\mathrm{Si}_{0}$ & $\mathrm{IIM}$ & Cal \\
$\mathrm{P}_{0}$ & $\mathrm{IIIM}$ & DolomíaC \\
$\mathrm{S}_{0}$ & $\mathrm{SL}$ & Sínter \\
$\mathrm{T}_{\mathrm{ARR}}$ & $\mathrm{SLI}$ & Chatarra \\
$\mathrm{ARR}$ & $\mathrm{SLF}$ & DolomíaCr \\
\hline
\end{tabular}

Variables finales: Variables dependientes

\begin{tabular}{|c|c|}
\hline $\begin{array}{c}\text { Composición } \\
\text { del acero }\end{array}$ & $\begin{array}{c}\text { Composición } \\
\text { de la escoria }\end{array}$ \\
\hline $\mathrm{C}_{\mathrm{f}}$ & $\mathrm{SiO}_{2}$ \\
$\mathrm{Si}_{\mathrm{f}}$ & $\mathrm{MnO}$ \\
$\mathrm{Mn}_{\mathrm{f}}$ & $\mathrm{P}_{2} \mathrm{O}_{5}$ \\
$\mathrm{P}_{\mathrm{f}}$ & $\mathrm{S}$ \\
$\mathrm{S}_{\mathrm{f}}$ & $\mathrm{TiO}_{2}$ \\
& $\mathrm{Fe}$ \\
& $\mathrm{CaO}$ \\
& $\mathrm{MgO}$ \\
& $\mathrm{Al}_{2} \mathrm{O}_{3}$ \\
\hline
\end{tabular}

\subsection{Variables dependientes}

\subsubsection{Composición final del acero}

- Porcentajes de carbono, manganeso, silicio, azufre y fósforo.

\subsubsection{Composición de la escoria}

- Porcentajes de $\mathrm{SiO}_{2}, \mathrm{MnO}, \mathrm{P}_{2} \mathrm{O}_{5}, \mathrm{~S}, \mathrm{TiO}_{2}, \mathrm{Fe}$, $\mathrm{CaO}, \mathrm{MgO}$ y $\mathrm{Al}_{2} \mathrm{O}_{3}$.

En la tabla II se muestran los valores medios, desviaciones estándar e intervalos máximos y mínimos para cada una de las variables analizadas. Se han estudiado los datos procedentes de 210 operaciones en procesos siderúrgicos L.B.E. Los pequeños valores obtenidos para las desviaciones reflejan 
TABLA II.- Análisis estadístico de los datos utilizados en los ajustes

TABLE II.- Statistical analysis of the data used in the fitting

\begin{tabular}{|c|c|c|c|c|}
\hline Variable & Media & $\begin{array}{l}\text { Int. } \\
\text { mín. }\end{array}$ & $\begin{array}{l}\text { Int. } \\
\text { máx. }\end{array}$ & $\begin{array}{l}\text { Desviación } \\
\text { estándar }\end{array}$ \\
\hline \multicolumn{5}{|c|}{ Composición del arrabio } \\
\hline $\mathrm{C}_{0}$ & 4,405 & 3,960 & 4,894 & 0,224 \\
\hline $\mathrm{Mn}_{0}$ & 0,581 & 0,454 & 0,784 & 0,085 \\
\hline $\mathrm{Si}_{0}$ & 0,922 & 0,607 & 1,279 & 0,165 \\
\hline $\mathrm{P}_{0}$ & 0,061 & 0,052 & 0,071 & $4,326 \mathrm{E}-3$ \\
\hline $\mathrm{S}_{0}$ & 0,011 & 0,007 & 0,019 & $2,75 \mathrm{E}-3$ \\
\hline \multicolumn{5}{|c|}{ Composición del acero } \\
\hline $\mathrm{C}_{\mathrm{f}}$ & 0,046 & 0,027 & 0,085 & 0,012 \\
\hline $\mathrm{Si}_{\mathrm{f}}$ & 0,005 & 0,001 & 0,008 & $1,137 \mathrm{E}-3$ \\
\hline$S_{f}$ & 0,012 & 0,008 & 0,021 & $2,693 \mathrm{E}-3$ \\
\hline$P_{f}$ & 0,012 & 0,008 & 0,130 & 0,017 \\
\hline $\mathrm{Mn}_{\mathrm{f}}$ & 0,156 & 0,090 & 0,268 & 0,036 \\
\hline $\mathrm{T}_{\mathrm{ARR}}$ & 1.288 & 1.180 & 1.375 & 37,52 \\
\hline \multicolumn{5}{|c|}{ Composición de la escoria } \\
\hline $\mathrm{SiO}_{2}$ & 17,92 & 15,13 & 21,83 & 1,658 \\
\hline $\mathrm{MnŌ}$ & 6,380 & 4,98 & 7,760 & 0,701 \\
\hline $\mathrm{P}_{2} \mathrm{O}_{5}$ & 1,270 & 1,040 & 1,630 & 0,138 \\
\hline $\mathrm{S}$ & 0,010 & 0 & 0,030 & $8,83 \mathrm{E}-3$ \\
\hline $\mathrm{TiO}_{2}$ & 0,700 & 0,5 & 0,900 & 0,078 \\
\hline $\mathrm{Fe}_{\mathrm{t}} \mathrm{O}$ & 14,90 & 10,50 & 24,30 & 2,956 \\
\hline $\mathrm{CaO}$ & 45,40 & 39,40 & 48,60 & 1,872 \\
\hline $\mathrm{MgO}$ & 6,100 & 5 & 6,900 & 0,470 \\
\hline $\mathrm{Al}_{2} \mathrm{O}_{3}$ & 0 & 0 & 9 & 1,260 \\
\hline \multicolumn{5}{|c|}{ Aditivos añadidos } \\
\hline Espato & 0,330 & 0 & 0,432 & 0,127 \\
\hline Precoque & 0 & 0 & 0,550 & 0,124 \\
\hline $\mathrm{Cal}$ & 9,145 & 7,125 & 10,98 & 0,929 \\
\hline DolomíaC & 3,697 & 0,679 & 5,948 & 0,633 \\
\hline Sinter & 3,252 & 0 & 7,074 & 1,823 \\
\hline CHT & 55,70 & 40,80 & 613 & 78,34 \\
\hline ARR & 220,2 & 15,80 & 447,9 & 43,55 \\
\hline \multicolumn{5}{|c|}{ Oxígeno soplado } \\
\hline ign & 22 & 0 & 313 & 47,38 \\
\hline IM & 961 & 885 & 1.156 & 63,73 \\
\hline IIM & 2.006 & 1.998 & 2.282 & 57,07 \\
\hline IIIM & 2.071 & 1.910 & 2.393 & 99,11 \\
\hline SL & 4.973 & 4.509 & 5.621 & 244,9 \\
\hline SLI & 1.776 & 1.116 & 2.681 & 328,8 \\
\hline SLF & 396 & 294 & 621 & 68,15 \\
\hline
\end{tabular}

que el control de las variables se encuentra dentro de límites muy estrictos.

Una vez seleccionadas las variables, se trata de determinar aquellas relaciones que permitan expli- car el comportamiento final de éstas, y así poder predecir en cada una de las operaciones los valores de composición del acero y de la escoria. Las relaciones obtenidas son ecuaciones de tipo multilineal (3 y 4$)$

$$
Y_{i}=\Sigma A_{i j} X_{j}
$$

Se ha verificado la validez de estas expresiones haciendo un estudio de los residuos, del coeficiente de regresión y de los valores predichos frente a los observados, como se muestra en las distintas relaciones obtenidas en los siguientes apartados. Se ha ajustado un total de 210 coladas de operación. Los coeficientes $A_{\mathrm{ij}}$ obtenidos para las distintas relaciones, utilizando operaciones caracterizadas por estrategias definidas de aditivos, oxígeno soplado y composición de arrabio, se recogen en la tabla III.

\section{DISCUSIÓN DE RESULTADOS}

\subsection{Ecuación para el carbono final}

La relación obtenida para el carbono en el acero es la siguiente:

$$
\begin{aligned}
& \mathrm{C}_{\mathrm{f}}=\mathrm{Si}_{0} * \mathrm{~A}_{\mathrm{C}_{\mathrm{f} / \mathrm{Si}_{0}}}+\mathrm{Mn}_{0} * \mathrm{~A}_{\mathrm{C}_{\mathrm{f} / \mathrm{Mn}}}+\mathrm{S}_{0} * \mathrm{~A}_{\mathrm{C}_{\mathrm{f} / \mathrm{S}_{0}}}+ \\
& + \text { DolomíaCr } * \mathrm{~A}_{\mathrm{C}_{\mathrm{f} / \mathrm{Dolomí}}}+\mathrm{Cal} * \mathrm{~A}_{\mathrm{C}_{\mathrm{f} / \mathrm{Cal}}}+ \\
& +\mathrm{ARR} * \mathrm{~A}_{\mathrm{C}_{\mathrm{f}_{/ \mathrm{ARR}}}}-\mathrm{IM} * \mathrm{~A}_{\mathrm{C}_{\mathrm{f} / \mathrm{IM}}}-\mathrm{IIM} * \mathrm{~A}_{\mathrm{C}_{\mathrm{f} / \mathrm{IIM}}}- \\
& -\mathrm{SL} * \mathrm{~A}_{\mathrm{C}_{\mathrm{f} / \mathrm{SL}}}-\mathrm{SLI} * \mathrm{~A}_{\mathrm{C}_{\mathrm{f} / \mathrm{SLI}}}-\mathrm{SLF} * \mathrm{~A}_{\mathrm{C}_{\mathrm{f} / \mathrm{SLF}}}
\end{aligned}
$$

\subsubsection{Efecto de la composición del arrabio}

Cantidades iniciales elevadas de silicio, manganeso y azufre perjudican la eliminación del carbono. La fisicoquímica del proceso permite predecir que la eliminación del carbono se realiza a través de la reacción acero-escoria

$$
\mathrm{C}+(\mathrm{FeO}) \rightarrow \mathrm{CO}(\mathrm{g})+\mathrm{Fe}
$$

en la que el carbono reacciona con el $\mathrm{FeO}$ de la escoria en la interfase metal/escoria formando $\mathrm{CO}$ y $\mathrm{CO}_{2}$ que se eliminan a través de los humos. Igualmente, las reacciones de eliminación del manganeso y del fósforo transcurren a través de reacciones similares vía escoria $(5$ y 6$)$ :

$$
\begin{gathered}
\mathrm{Mn}+(\mathrm{FeO}) \rightarrow(\mathrm{MnO})+\mathrm{Fe} \\
2 \mathrm{P}+5(\mathrm{FeO})+(\mathrm{CaO}) \rightarrow \mathrm{Ca}_{3}\left(\mathrm{PO}_{4}\right)_{2}
\end{gathered}
$$


TABLA III.- Coeficientes $\mathrm{A}_{\mathrm{ij}}$ correspondientes a las relaciones obtenidas para cada una de las variables dependientes consideradas

TABLE III.- $\mathrm{A}_{\mathrm{ij}}$ coefficients in the obtained relationships for each one of the considered dependent variables

\begin{tabular}{|c|c|c|c|c|c|c|c|c|c|c|c|}
\hline & $A_{i j}$ & $\mathrm{C}_{\mathrm{f}}$ & $\mathrm{Mn}_{\mathrm{f}}$ & $\mathrm{P}_{2} \mathrm{O}_{5}$ & $\mathrm{MnO}$ & $\mathrm{Fe}_{\mathrm{t}} \mathrm{O}$ & $\mathrm{CaO}$ & $\mathrm{SiO} 2$ & DolomíaC & $\mathrm{CHT}$ & $\mathrm{Cal}$ \\
\hline Arrabio & $\begin{array}{l}\mathrm{C}_{0} \\
\mathrm{Mn}_{0} \\
\mathrm{Si}_{0} \\
\mathrm{~S}_{0} \\
\mathrm{P}_{0} \\
\mathrm{ARR} \\
\mathrm{T}_{\mathrm{ARR}}\end{array}$ & $\begin{array}{l}0,095 \\
0,016 \\
0,67 \\
0,001\end{array}$ & $\begin{array}{l}0,2 \\
0,9 \\
0,001 \\
4,6 \mathrm{E}-4\end{array}$ & $\begin{array}{l}0,6 \\
3,6 \\
0,01 \\
4,7 \mathrm{E}-4\end{array}$ & $\begin{array}{l}4 \\
8,5 \\
0,03\end{array}$ & $\begin{array}{r}3,29 \\
\\
3,25 \\
132,4\end{array}$ & $\begin{array}{l}8,3 \\
\\
0,1 \\
0,031\end{array}$ & $\begin{array}{l}3 \\
5,1 \\
\\
0,06 \\
0,02\end{array}$ & $\begin{array}{l}0,6 \\
3,2 \\
7,9 \\
0,02\end{array}$ & $\begin{array}{c}25,1 \\
218,3 \\
78 \\
0,02\end{array}$ & $\begin{array}{c}2 \\
\\
5,6 \\
69,8 \\
31,9 \\
0,06 \\
0,007\end{array}$ \\
\hline Adicionantes & $\begin{array}{l}\text { Espato } \\
\text { Precoque } \\
\text { Cal } \\
\text { DolomíaC } \\
\text { DolomíaCr } \\
\text { Sínter } \\
\text { CHT }\end{array}$ & $\begin{array}{l}0,005 \\
0,001\end{array}$ & $\begin{array}{l}0,03 \\
0,003 \\
\\
0,006 \\
0,01 \\
0,003\end{array}$ & $\begin{array}{l}0,04 \\
0,013 \\
0,0055\end{array}$ & $\begin{array}{l}0,6 \\
0,333 \\
0,09 \\
0,06 \\
0,04\end{array}$ & $\begin{array}{l}6,5 \\
0,6 \\
0,69 \\
0,4 \\
0,6 \\
0,1\end{array}$ & $\begin{array}{l}1,6 \\
\\
0,4 \\
0,6\end{array}$ & $\begin{array}{l}0,3 \\
0,2\end{array}$ & & 0,7 & \\
\hline Soplado & $\begin{array}{l}\text { ign } \\
\text { IM } \\
\text { IIM } \\
\text { IIIM } \\
\text { SL } \\
\text { SLI } \\
\text { SLF }\end{array}$ & $\begin{array}{l}3,7 \mathrm{E}-5 \\
5,5 \mathrm{E}-5 \\
\\
3,2 \mathrm{E}-5 \\
1,8 \mathrm{E}-5 \\
1,9 \mathrm{E}-5\end{array}$ & $\begin{array}{l}4,8 \mathrm{E}-5 \\
1 \mathrm{E}-4 \\
\\
6 \mathrm{E}-5 \\
5 \mathrm{E}-5 \\
4,9 \mathrm{E}-5\end{array}$ & $\begin{array}{l}3,8 \mathrm{E}-4 \\
1,2 \mathrm{E}-4 \\
1,1 \mathrm{E}-4 \\
1,5 \mathrm{E}-4\end{array}$ & $\begin{array}{l}5,2 \mathrm{E}-4 \\
6,9 \mathrm{E}-4 \\
\\
2,2 \mathrm{E}-4 \\
3,3 \mathrm{E}-4\end{array}$ & $\begin{array}{l}0,003 \\
\\
0,002 \\
0,005 \\
0,005\end{array}$ & $\begin{array}{l}0,004 \\
0,002 \\
0,004 \\
0,0037\end{array}$ & $\begin{array}{l}0,002 \\
\\
0,003 \\
0,002 \\
0,001\end{array}$ & $1,4 \mathrm{E}-4$ & $\begin{array}{l}0,006 \\
0,011 \\
\\
0,006 \\
0,007 \\
0,005\end{array}$ & $\begin{array}{l}0,007 \\
0,002 \\
0,001\end{array}$ \\
\hline $\begin{array}{l}\text { Coeficiente } \\
\text { de regresión }\end{array}$ & & 0,94 & 0,98 & 0,99 & 0,99 & 0,98 & 0,99 & 0,99 & 0,98 & 0,99 & 0,99 \\
\hline
\end{tabular}

El manganeso presentará una competencia hacia el consumo del FeO de la escoria para la eliminación del carbono. Por otra parte, el silicio del arrabio se elimina durante los primeros momentos del soplado por acción directa del chorro de oxígeno:

$$
\mathrm{Si}+2 \mathrm{O} \rightarrow\left(\mathrm{SiO}_{2}\right)
$$

Cuanto mayor sea la cantidad de silicio tanto más tardará en transcurrir esta reacción, pudiendo tener lugar:

$$
\mathrm{Si}+2(\mathrm{FeO}) \rightarrow\left(\mathrm{SiO}_{2}\right)+2 \mathrm{Fe}
$$

que consumiría parte del $\mathrm{FeO}$ perjudicando la eliminación del carbono.

El hecho de que el silicio se oxide con gran rapidez no excluye una cierta oxidación del carbono simultáneamente (5). La tendencia a repartirse el oxígeno entre ambos modelos viene dada por la diferencia de entalpía libre liberada en cada una de las siguientes reacciones:

$$
\mathrm{O}+\mathrm{Si} \rightarrow\left(\mathrm{SiO}_{2}\right)_{\beta-\text { cristal }}
$$

$$
\mathrm{C}+\mathrm{O} \rightarrow \mathrm{CO}(\mathrm{g})
$$

\subsubsection{Influencia del patrón de soplado}

El oxígeno soplado durante el proceso de afino va a beneficiar la eliminación del carbono final. Takawa y col. (7) consideraron que el responsable de la oxidación del carbono es el oxígeno soplado durante el afino. Se observa, de acuerdo con la ec. [1], que el oxígeno soplado a lo largo del proceso de afino beneficia siempre la eliminación del carbono.

\subsubsection{Influencia de los aditivos}

Concentraciones elevadas de cal y dolomía cruda perjudican la eliminación del carbono, ya que afectan a la naturaleza de la emulsión metal-escoria que influye en la eliminación del carbono por reacción con el $\mathrm{FeO}$ que forma parte del contenido de la escoria.

En la figura 1 se han representado los valores observados frente a los predichos para el carbono. El ajuste tiene un coeficiente de regresión de 0,94. 


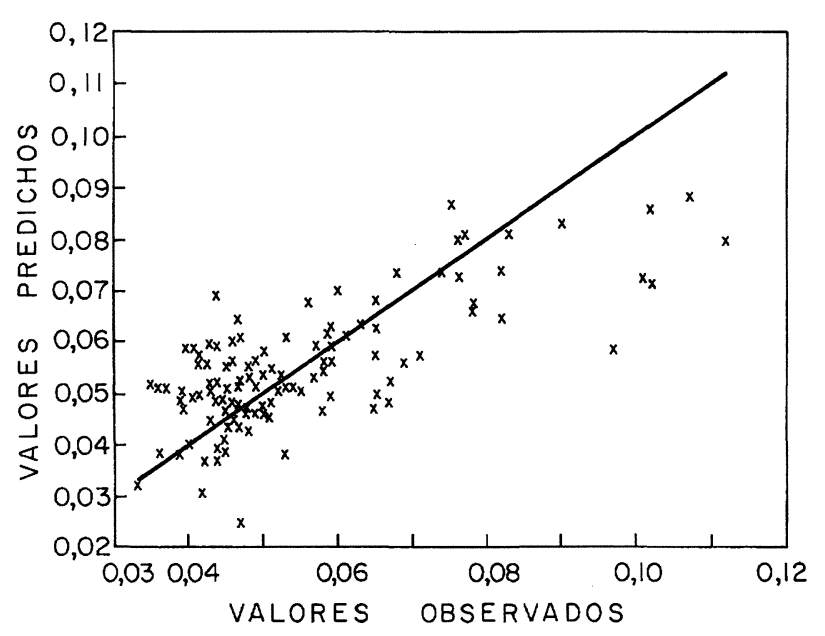

FIG. 1.- Valores predichos frente a valores observados para la determinación de la concentración final del carbono.

Fig. 1.- Predicted values vs. observed values of final carbon concentration.

\subsection{Ecuación para la concentración final de manganeso}

La reacción para la eliminación del manganeso es la siguiente:

$$
\begin{aligned}
& \mathrm{Mn}_{\mathrm{f}}=\mathrm{S}_{0} * \mathrm{~A}_{\mathrm{Mn}_{\mathrm{f}} / \mathrm{S}_{0}}-\text { Espato } * \mathrm{~A}_{\mathrm{Mn}_{\mathrm{f} / \text { Espato }}}+ \\
& +\mathrm{Cal} * \mathrm{~A}_{\mathrm{Mn}_{\mathrm{f} / \mathrm{Cal}}}+\mathrm{Mn}_{0} * \mathrm{~A}_{\mathrm{Mn}_{\mathrm{f} / \mathrm{Mn}_{0}}}- \\
& \text { - DolomíaCr* } \mathrm{A}_{\mathrm{Mnf}_{\mathrm{f} / \text { DolomíaCr }}} \text { - Sínter* } \mathrm{A}_{\mathrm{Mnf}_{\mathrm{f} / \text { Sínter }}}- \\
& -\mathrm{CHT} * \mathrm{~A}_{\mathrm{Mnf}_{\mathrm{f}} \mathrm{CHT}}+\mathrm{ARR} * \mathrm{~A}_{\mathrm{Mnf}_{\mathrm{ARR}}}-\mathrm{IM} * \mathrm{~A}_{\mathrm{Mnf}_{\mathrm{f} / \mathrm{M}}}- \\
& -\mathrm{IIM} * \mathrm{~A}_{\mathrm{Mn}_{\mathrm{f} / \mathrm{IIM}}}-\mathrm{SL} * \mathrm{~A}_{\mathrm{Mn}_{\mathrm{f} / \mathrm{SL}}}-\mathrm{SLI} * \mathrm{~A}_{\mathrm{Mn}_{\mathrm{f} / \mathrm{SLI}}}- \\
& -\mathrm{SLF} * \mathrm{~A}_{\mathrm{Mn}_{\mathrm{f} / \mathrm{SLF}}}+\mathrm{T}_{\mathrm{ARR}} * \mathrm{~A}_{\mathrm{Mn}_{\mathrm{f}} / \mathrm{T}_{\mathrm{ARR}}}
\end{aligned}
$$

$$
(\mathrm{FeO})+\mathrm{Mn} \Leftrightarrow(\mathrm{MnO})+\mathrm{Fe}
$$

La eliminación del manganeso se caracteriza porque al principio su tendencia a la escorificación es elevada (8), ya que la masa de la escoria y su contenido de $\mathrm{FeO}$ aumenta rápidamente. Hacia la mitad del afino, el FeO disminuye en la escoria y la temperatura se estabiliza, dando lugar a una reversión del manganeso a la fase metal. Al final del soplado un aumento rápido del $\mathrm{FeO}$ permite una nueva oxidación. La reacción hacia la derecha se favorece con un gran volumen de escoria, baja temperatura y alto porcentaje de $\mathrm{FeO}$ en la escoria. $\mathrm{La}$ reacción hacia la izquierda se favorece con temperatura elevada y pequeños porcentajes de $\mathrm{FeO}$.

\subsubsection{Influencia del arrabio}

Concentraciones elevadas de manganeso y de azufre en el arrabio perjudican la eliminación del manganeso. Las reacciones para la eliminación del azufre podrían tener lugar a través de las siguientes reacciones:

$$
\begin{aligned}
& (\mathrm{CaO})+\mathrm{FeS} \rightarrow(\mathrm{CaS})+(\mathrm{FeO}) \\
& (\mathrm{CaO})+\mathrm{MnS} \rightarrow(\mathrm{CaS})+(\mathrm{MnO})
\end{aligned}
$$

Considerando que la eliminación del azufre ocurre de acuerdo con la ec. [12], ésta da lugar a la formación de $\mathrm{MnO}$. Cantidades elevadas de $\mathrm{MnO}$ producen un desplazamiento de la reacción de eliminación del manganeso hacia la izquierda; es decir, hacia la formación del manganeso en el acero [10], incrementando la concentración final de manganeso; por otra parte, concentraciones elevadas de azufre necesitan incrementar la concentración inicial de cal para su eliminación, situación que perjudica la concentración final de manganeso. Elevadas temperaturas iniciales del arrabio perjudican la eliminación del manganeso. De acuerdo con la siguiente expresión, se puede observar cómo el coeficiente de reparto disminuye cuando aumenta la temperatura del baño:

$$
\log \frac{(\mathrm{MnO})}{\mathrm{Mn}}=\frac{5.548}{\mathrm{~T}}-3,87
$$

\subsubsection{Influencia del patrón de soplado}

El oxígeno soplado durante el proceso beneficia la eliminación del manganeso.

\subsubsection{Influencia de los aditivos}

Los aditivos añadidos favorecen, en general, la eliminación del manganeso a excepción de la cal formadora de escoria. Suito y col. (9 y 10) estudiaron la variación de la constante de equilibrio para distintas basicidades, encontrando que, a medida que aumenta la basicidad de la escoria, disminuye la constante de equilibrio y, por tanto, la cantidad de manganeso eliminado. Esta basicidad aumenta a medida que aumenta la concentración de $\mathrm{CaO}$ en la escoria, de acuerdo con la siguiente expresión:

$$
\mathrm{B}=\frac{\% \mathrm{CaO}+0,3 * \% \mathrm{MgO}}{\% \mathrm{SiO}_{2}+\% \mathrm{P}_{2} \mathrm{O}_{5}}
$$




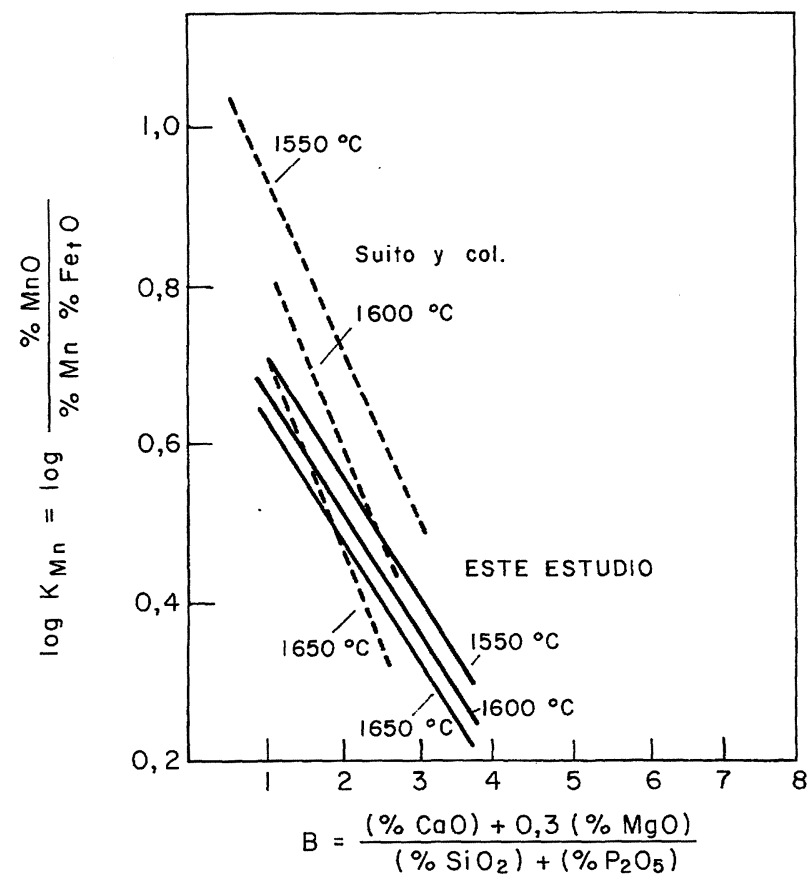

FIG. 2.- Variación de la constante de equilibrio del manganeso con la basicidad de la escoria para distintas temperaturas.

Fig. 2.- Manganese equilibrium constant vs. the basicity of the slag at different temperatures.

Los valores de la constante de equilibrio se analizaron, como una función de la composición de la escoria, por un método de regresión múltiple. En la figura 2 se muestran las tendencias de la constante para distintas temperaturas comparando los datos analizados por Suito y col. (9) con los analizados para escorias procedentes de convertidores tipo L.B.E. (ENSIDESA).

Adiciones de sínter benefician la eliminación del manganeso, ya que el desplazamiento del equilibrio hacia la formación de $\mathrm{MnO}$ se favorece cuando se tienen cantidades elevadas de $\mathrm{FeO}$ para bajas temperaturas, y el sínter aporta por una parte mineral de hierro y por otra actúa como refrigerante. Igual efecto producen las adiciones de chatarra.

En la figura 3 se representan los valores predichos frente a los observados para el manganeso, obteniéndose un coeficiente de regresión de 0,98 .

Igualmente, se realizaron ajustes del azufre y del fósforo en el acero; sin embargo, los resultados obtenidos no son aceptables, ajustándose posteriormente los valores de la composición de la escoria que se pueden relacionar con las concentraciones iniciales del acero. Resultaron relaciones interesantes para el $\mathrm{SiO}_{2}, \mathrm{P}_{2} \mathrm{O}_{5}, \mathrm{MgO}, \mathrm{Fe}, \mathrm{CaO}$ y $\mathrm{MnO}$ de la escoria.

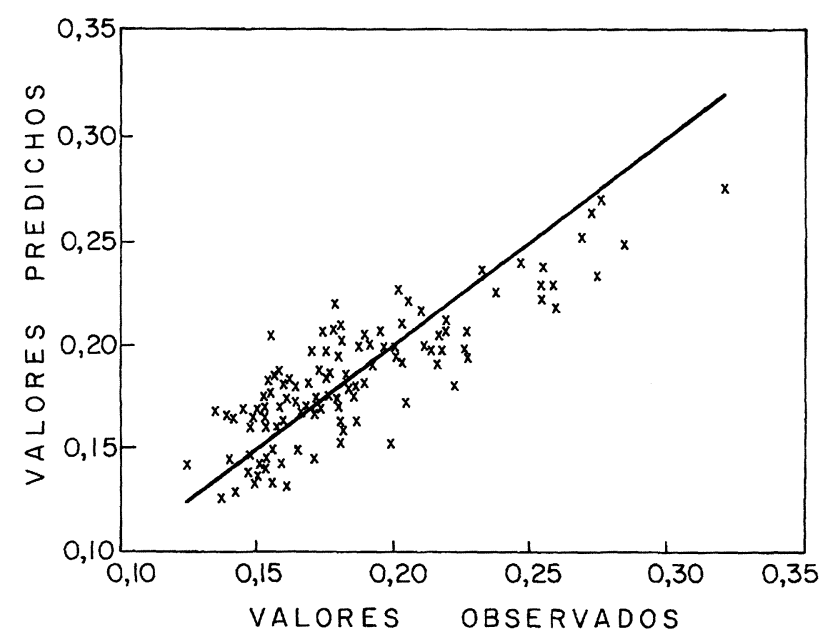

FIG. 3.- Valores predichos frente a valores observados para la determinación de la concentración final del manganeso.

Fig. 3.- Predicted values vs. observed values of manganese final concentration.

\subsection{Ecuación para el $\mathrm{P}_{2} \mathrm{O}_{5}$ en la escoria}

$$
\begin{aligned}
& \mathrm{P}_{2} \mathrm{O}_{5}=-\mathrm{Si}_{0} * \mathrm{~A}_{\mathrm{P}_{2} \mathrm{O}_{5 / \mathrm{Si} 0}}+\mathrm{P}_{0} * \mathrm{~A}_{\mathrm{P}_{2} \mathrm{O}_{5 / \mathrm{P}_{0}}}- \\
& \text { - DolomíaC* } \mathrm{A}_{\mathrm{P}_{2} \mathrm{O}_{5 / \text { DolomíaC }}}-\text { Sínter* } \mathrm{A}_{\mathrm{P}_{2} \mathrm{O}_{5 / \text { Sínter }}}+
\end{aligned}
$$

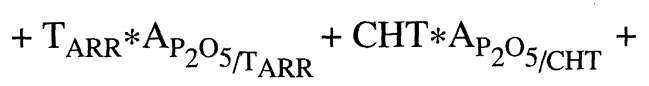

$$
\begin{aligned}
& +\mathrm{ARR} * \mathrm{~A}_{\mathrm{P}_{2} \mathrm{O}_{5 / \mathrm{ARR}}}-\mathrm{IIM} * \mathrm{~A}_{\mathrm{P}_{2} \mathrm{O}_{5 / \mathrm{IIM}}}- \\
& -\mathrm{SL} * \mathrm{~A}_{\mathrm{P}_{2} \mathrm{O}_{5 / \mathrm{SL}}}-\mathrm{SLI} * \mathrm{~A}_{\mathrm{P}_{2} \mathrm{O}_{5 / \mathrm{SLI}}}-\mathrm{SLF} * \mathrm{~A}_{\mathrm{P}_{2} \mathrm{O}_{5 / \mathrm{SLF}}}
\end{aligned}
$$

\subsubsection{Influencia de la composición del arrabio}

Concentraciones elevadas de fósforo dan lugar a porcentajes de $\mathrm{P}_{2} \mathrm{O}_{5}$ elevados [16] y [17]. Concentraciones elevadas de silicio perjudican la eliminación del fósforo, debido a las reacciones del silicio con el oxígeno soplado y la cal añadida [18]. El consumo de cal, por parte de la reacción del silicio, impide fijar el fósforo en la escoria cuya fijación evitaría su desplazamiento desde la fase escoria hacia la fase metal (11-13).

$$
\begin{gathered}
2(\mathrm{FeO})+2 \mathrm{P} \rightarrow 5 \mathrm{Fe}+\left(\mathrm{P}_{2} \mathrm{O}_{5}\right) \\
3(\mathrm{CaO})+\left(\mathrm{P}_{2} \mathrm{O}_{5}\right) \rightarrow \mathrm{Ca}_{3}\left(\mathrm{PO}_{4}\right)_{2} \\
\mathrm{Si}+(\mathrm{FeO})+(\mathrm{CaO}) \rightarrow \mathrm{SiO}_{2} \mathrm{CaO}+\mathrm{Fe}
\end{gathered}
$$




\subsubsection{Influencia del patrón de soplado}

De acuerdo con el ajuste, cuando se aumenta la cantidad de oxígeno soplado disminuye el porcentaje de $\mathrm{P}_{2} \mathrm{O}_{5}$ en la escoria, ya que pueden tener lugar reacciones de competencia para la eliminación del silicio, carbono y manganeso que consumen oxígeno a través de la formación de $\mathrm{FeO}$.

$$
\mathrm{Fe}+\mathrm{C} \rightarrow(\mathrm{FeO})
$$

\subsubsection{Influencia de los aditivos}

Las adiciones de chatarra benefician esta eliminación, mientras que el sínter y la dolomía añadidos la perjudican. Las adiciones de chatarra actúan como refrigerante para el balance térmico, condición que favorece la eliminación del fósforo. La ecuación no predice adecuadamente el efecto del sínter, ya que altos contenidos de $\mathrm{FeO}$ para bajas temperaturas benefician la formación de $\mathrm{P}_{2} \mathrm{O}_{5}$ que se consigue añadiendo mineral de hierro.

En la figura 4 se representan los valores predichos frente a los observados para el $\mathrm{P}_{2} \mathrm{O}_{5}$ de la escoria, obteniéndose un coeficiente de regresión de 0,99.

\subsection{Ecuación para el $\mathrm{MnO}$ en la escoria}

$$
\begin{aligned}
& \mathrm{MnO}=- \text { Espato }^{2} \mathrm{~A}_{\mathrm{MnO}}{ }_{\text {/Espato }}-\mathrm{SLI} * \mathrm{~A}_{\mathrm{MnO}_{/ \mathrm{SLI}}}-
\end{aligned}
$$

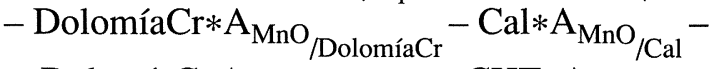

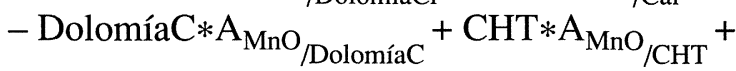

$$
\begin{aligned}
& +\mathrm{ARR} * \mathrm{~A}_{\mathrm{MnO} / \mathrm{ARR}}-\mathrm{IIM} * \mathrm{~A}_{\mathrm{MnO}}{ }_{/ \mathrm{IIM}}+
\end{aligned}
$$

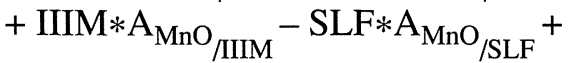

$$
\begin{aligned}
& +\mathrm{Mn}_{\mathrm{O}} * \mathrm{~A}_{\mathrm{MnO}_{/ \mathrm{MnO}}}-\mathrm{P}_{0} * \mathrm{~A}_{\mathrm{MnO}} \mathrm{P}_{0}
\end{aligned}
$$

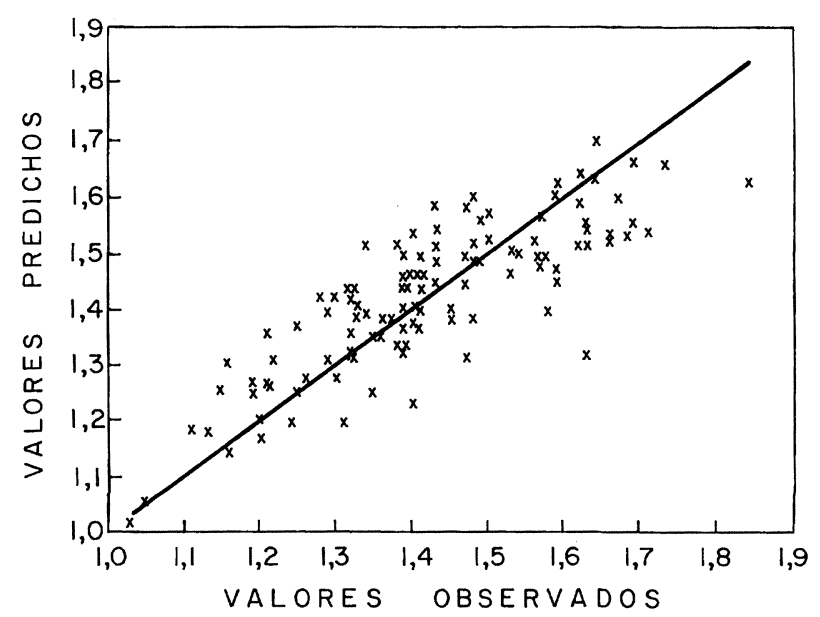

FIG. 4.- Valores predichos frente a valores observados para el contenido de $\mathrm{P}_{2} \mathrm{O}_{5}$ de la escoria.

Fig. 4.- Predicted values vs. observed values of $\mathrm{P}_{2} \mathrm{O}_{5}$ in the slag.

\subsubsection{Influencia de la composición del arrabio}

Concentraciones elevadas de manganeso favorecen su eliminación aumentando la concentración de su óxido correspondiente en la escoria, mientras que la presencia de fósforo la perjudican, ya que la eliminación del fósforo se favorece con altos contenidos de $\mathrm{FeO}$ y con el aumento del índice de basicidad; es decir, aumento de cal añadida a la escoria, situación que perjudica la formación de $\mathrm{MnO}$ de acuerdo con la ec. [20].

\subsubsection{Influencia del patrón de soplado}

El oxígeno soplado correspondiente a IIIM es el único que favorece la formación del $\mathrm{MnO}$.

\subsubsection{Influencia de los aditivos}

Los aditivos añadidos perjudican la formación de $\mathrm{MnO}$, excepto la chatarra y el arrabio. Cuando aumenta la cantidad de dolomía y cal se produce un incremento de la basicidad que perjudica la eliminación del manganeso y, por tanto, la formación de $\mathrm{MnO}$.

En la figura 5 se representan los valores predichos frente a los observados para el $\mathrm{MnO}$, siendo el coeficiente de regresión de 0,99.

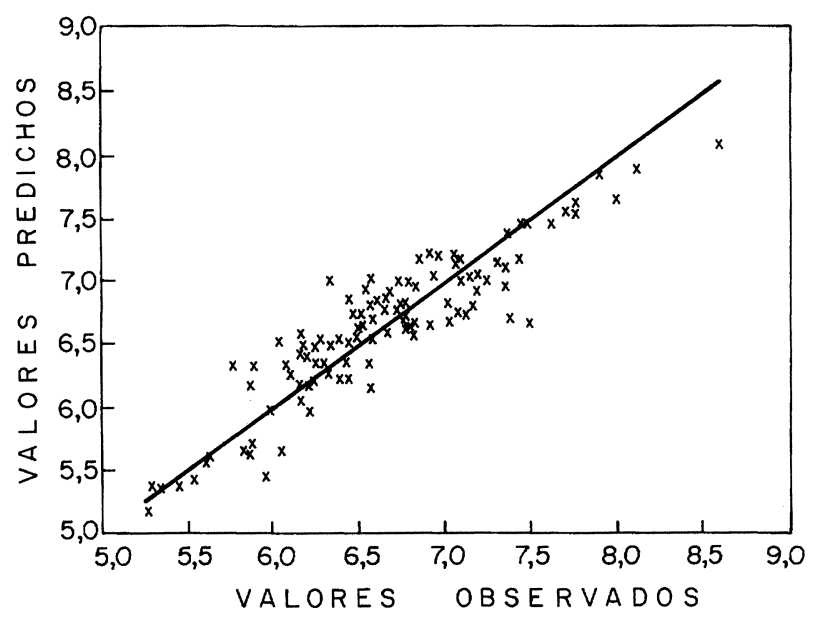

FIG. 5.- Valores predichos frente a valores observados para el contenido de $\mathrm{MnO}$ de la escoria.

Fig. 5.-Predicted values vs. observed values of $\mathrm{MnO}$ in the slag. 


\subsection{Ecuación para el óxido de hierro total en la escoria}

$$
\begin{aligned}
& \mathrm{Fe}_{\mathrm{t}} \mathrm{O}=\text { Espato } * \mathrm{~A}_{\mathrm{Fe}_{\mathrm{t}} \mathrm{O} / \text { Espato }}-\mathrm{Cal} * \mathrm{~A}_{\mathrm{Fe}_{\mathrm{t}} \mathrm{O} / \mathrm{Cal}}+ \\
& + \text { Sínter } * \mathrm{~A}_{\mathrm{Fe}_{\mathrm{t}} \mathrm{O}_{/ \text {Sínter }}}+\mathrm{CHT} * \mathrm{~A}_{\mathrm{Fe}_{\mathrm{t}} \mathrm{O} / \mathrm{CHT}}+ \\
& +\mathrm{IIM} * \mathrm{~A}_{\mathrm{Fe}_{\mathrm{t}} \mathrm{O} / \mathrm{IIM}}+\mathrm{SL} * \mathrm{~A}_{\mathrm{Fe}_{\mathrm{t}} \mathrm{O} / \mathrm{SL}}+\mathrm{SLI} * \mathrm{~A}_{\mathrm{Fe}_{\mathrm{t}} \mathrm{O}_{/ \mathrm{SLI}}}+ \\
& +\mathrm{SLF} * \mathrm{~A}_{\mathrm{Fe}_{\mathrm{t}} \mathrm{O} / \mathrm{SLF}}-\mathrm{C}_{0} * \mathrm{~A}_{\mathrm{Fe}_{\mathrm{t}} \mathrm{O} / \mathrm{C}_{0}}-\mathrm{Si}_{0} * \mathrm{~A}_{\mathrm{Fe}_{\mathrm{t}} \mathrm{O}_{/ \mathrm{Si}_{0}}}- \\
& -\mathrm{S}_{0} * \mathrm{~A}_{\mathrm{Fe}_{\mathrm{t}} \mathrm{O}_{/ \mathrm{S}_{0}}}
\end{aligned}
$$

\subsubsection{Influencia de la composición del arrabio}

Concentraciones de silicio elevadas perjudican la formación de $\mathrm{FeO}$, ya que es un elemento consumidor de FeO, de acuerdo con la ec. [6]. Los ajustes no permiten predecir adecuadamente el efecto del azufre inicial, debido a que concentraciones elevadas de éste producen un desplazamiento de su equilibrio hacia la formación de $\mathrm{FeO}$ en la escoria según la ec. [11]; sin embargo, la reacción del azufre puede tener lugar a través de la reacción [12] y, por tanto, el efecto del $\mathrm{FeO}$ no quedaría reflejado en la expresión de ajuste; no obstante, la reacción de eliminación de azufre prácticamente no tiene lugar e, incluso, puede llegar a aumentar en función de los aditivos añadidos. Por otra parte, la eliminación del azufre, de acuerdo con las reacciones propuestas se favorece cuando existe suficiente cal libre, situación que perjudica la formación de $\mathrm{FeO}$ en la escoria según la ecuación propuesta.

\subsubsection{Influencia del patrón de soplado}

El oxígeno soplado favorece la formación de óxido de hierro, sobre todo en los momentos finales de la operación, ya que en esta etapa casi todas las impurezas han sido eliminadas y el oxígeno reaccionará preferentemente con el hierro, dando lugar a la formación del FeO.

\subsubsection{Influencia de los aditivos}

La presencia de cal perjudica esta formación, favoreciendo el resto de los aditivos la formación de óxidos de hierro en la escoria.

En la figura 6 se representan los valores predichos frente a los observados para el hierro total en la escoria, obteniéndose un coeficiente de regresión de 0,98 .

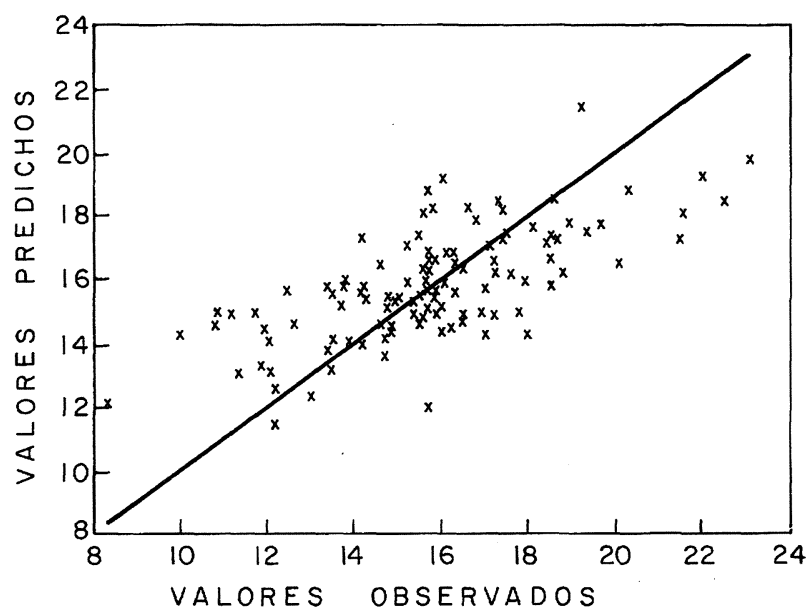

FIG. 6.- Valores predichos frente a valores observados para el contenido de óxido de hierro en la escoria.

Fig. 6.- Predicted values vs. observed values of $\mathrm{FeO}$ in the slag.

\subsection{Ecuación para el $\mathrm{CaO}$ en la escoria}

$$
\begin{aligned}
& \mathrm{CaO}=- \text { DolomíaCr } * \mathrm{~A}_{\mathrm{CaO}}{ }_{/ \text {DolomíaCr }}+\mathrm{Cal} * \mathrm{~A}_{\mathrm{CaO}} / \mathrm{Cal} \\
& - \text { Sínter } * \mathrm{~A}_{\mathrm{CaO}}{ }_{\text {/Sínter }}+\mathrm{ARR} * \mathrm{~A}_{\mathrm{CaO}} \mathrm{ARR}_{\text {ARR }}- \\
& -\mathrm{IIM} * \mathrm{~A}_{\mathrm{CaO}}{ }_{/ \mathrm{IIM}}-\mathrm{SL} * \mathrm{~A}_{\mathrm{CaO}}{ }_{/ \mathrm{SL}}-\mathrm{SLI} * \mathrm{~A}_{\mathrm{CaO} / \mathrm{SLI}}- \\
& -\mathrm{SLF} * \mathrm{~A}_{\mathrm{CaO}_{/ \mathrm{SLF}}}-\mathrm{Si}_{0} * \mathrm{~A}_{\mathrm{CaO}_{/ \mathrm{Si}_{0}}}+\mathrm{T}_{\mathrm{ARR}} * \mathrm{~A}_{\mathrm{CaO}}{ }_{\mathrm{T}_{\mathrm{ARR}}}
\end{aligned}
$$

\subsubsection{Influencia de la composición del arrabio}

De acuerdo con el ajuste, la única variable que parece tener influencia en la concentración de $\mathrm{CaO}$ es el silicio. Concentraciones elevadas de este elemento perjudican la presencia de cal libre en la escoria, ya que esta quedaría fijada formando silicato de calcio (ec. [18]).

\subsubsection{Influencia del patrón de soplado}

Cantidades elevadas de oxígeno soplado favorecen la oxidación de impurezas y, por tanto, su fijación en la cal añadida, disminuyendo, en este caso, también la concentración de cal libre presente en la escoria.

\subsubsection{Influencia de los aditivos}

Adiciones de cal y sínter benefician esta presencia, mientras que concentraciones de dolomía cruda la perjudican. 


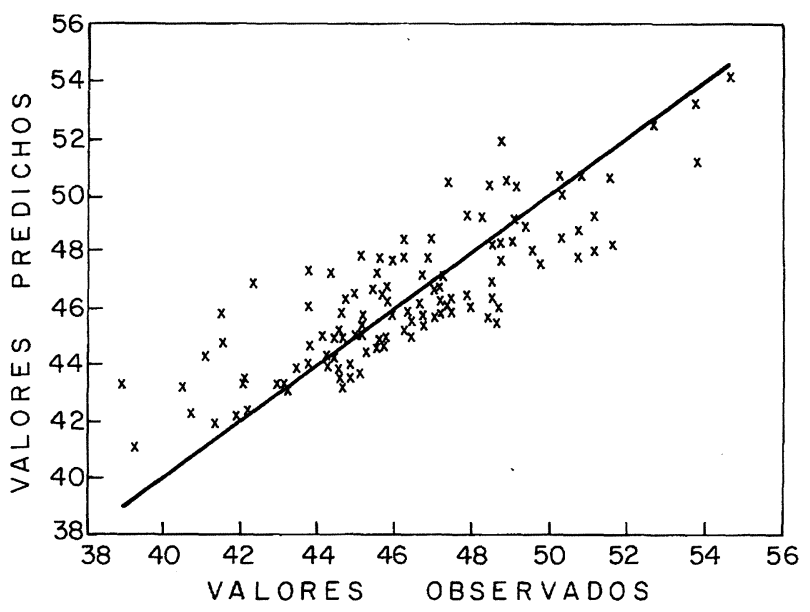

FIG. 7.- Valores predichos frente a valores observados para el contenido de $\mathrm{CaO}$ en la escoria.

Fig. 7.- Predicted values vs. observed values of $\mathrm{CaO}$ in the slag.

En la figura 7 se representan los valores predichos frente a los observados para el $\mathrm{CaO}$, obteniéndose un coeficiente de regresión de 0,99.

\subsection{Ecuación para el $\mathrm{SiO}_{2}$ en la escoria}

$$
\begin{aligned}
& \mathrm{SiO}_{2}=-\mathrm{C}_{0} * \mathrm{~A}_{\mathrm{SiO}_{2 / \mathrm{C}}}+\mathrm{Si}_{0} * \mathrm{~A}_{\mathrm{SiO}_{2 / \mathrm{Si}}}- \\
& -\mathrm{IIM} * \mathrm{~A}_{\mathrm{SiO}_{2 / \mathrm{IIM}}}-\mathrm{SL} * \mathrm{~A}_{\mathrm{SiO}_{2 / \mathrm{SL}}}-\mathrm{SLI} * \mathrm{~A}_{\mathrm{SiO}_{2 / \mathrm{SLI}}}{ }^{-} \\
& -\mathrm{SLF} * \mathrm{~A}_{\mathrm{SiO}_{2 / \mathrm{SLF}}}-\text { Espato*} \mathrm{A}_{\mathrm{SiO}_{2 / \mathrm{Espato}}}- \\
& - \text { DolomíaCr} * \mathrm{~A}_{\mathrm{SiO}_{2} / \mathrm{DolomíaCr}}-\text { Sínter } * \mathrm{~A}_{\mathrm{SiO}_{2 / \text { Sínter }}}+ \\
& +\mathrm{ARR} * \mathrm{~A}_{\mathrm{SiO}_{2 / \mathrm{ARR}}}+\mathrm{T}_{\mathrm{ARR}} * \mathrm{~A}_{\mathrm{SiO}_{2} / \mathrm{T}_{\mathrm{ARR}}}
\end{aligned}
$$

\subsubsection{Influencia de la composición del arrabio}

Concentraciones altas de silicio incrementan la concentración final de sílice en la escoria, perjudicando la presencia de carbono que actúa como elemento competidor y, por tanto, disminuye la eliminación del silicio (14). La oxidación del silicio ocurre muy rápidamente, en especial a bajas temperaturas. La reacción puede tener lugar directamente por acción del oxígeno soplado o bien a través del $\mathrm{FeO}$ formado en los últimos momentos de su eliminación; por ello, la presencia de otros elementos como el carbono puede perjudicar ésta, ya que la eliminación del carbono se puede considerar que ocurre a través de dos mecanismos. Chaterjee y col. (15) han estudiado la aportación a la eliminación del carbono, observando que, durante los primeros momentos, cuando el porcentaje de oxígeno soplado es pequeño, tiene lugar la descarburación

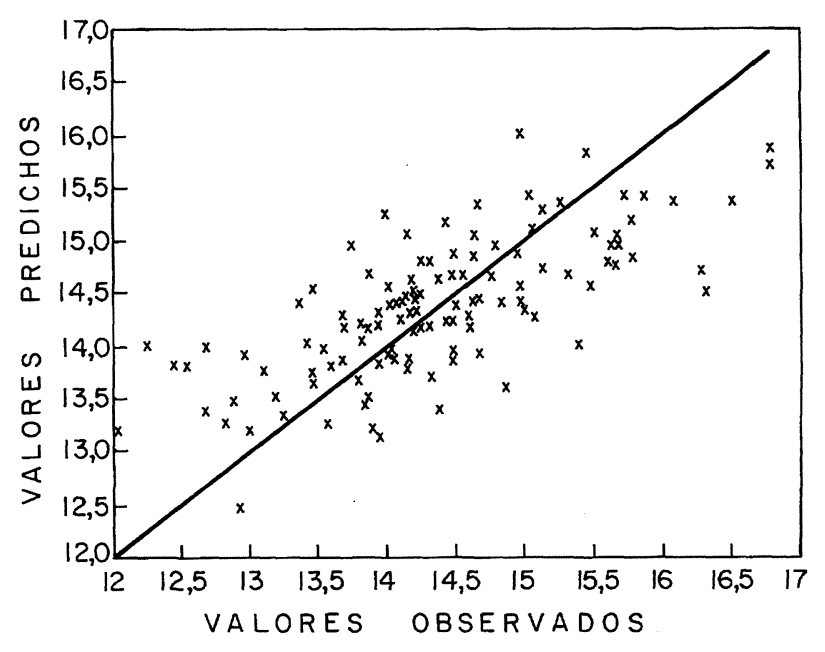

FIG. 8.- Valores predichos frente a valores observados para el contenido de $\mathrm{SiO}_{2}$ en la escoria.

Fig. 8.- Predicted values vs. observed values of $\mathrm{SiO}_{2}$ in the slag.

directa por acción del chorro de oxígeno (reacción [8]) y, posteriormente, la reacción tiene lugar de acuerdo con [2].

\subsubsection{Influencia del patrón de soplado}

El oxígeno soplado durante los momentos finales perjudica el porcentaje de silicio final en la escoria.

\subsubsection{Influencia del los aditivos}

El espato, la dolomía cruda y el sínter añadidos disminuyen el porcentaje de $\mathrm{SiO}_{2}$, mientras que el arrabio añadido favorece este porcentaje.

En la figura 8 se representan valores predichos frente a los observados para el $\mathrm{SiO}_{2}$, obteniéndose un coeficiente de regresión de 0,99 .

\subsection{Relaciones para los aditivos añadidos}

En los apartados anteriores se han obtenido relaciones para las variables dependientes: composición del acero y escoria, en función de las variables independientes. En este apartado se trata de obtener alguna expresión que permita predecir las cantidades de aditivos que se deben utilizar para obtener unos productos finales deseados a partir de la materia prima inicial, es decir, el arrabio procedente de hornos altos. En este estudio se considera que los productos finales de composición del acero corresponden a los detallados en la tabla II, de acuerdo con los valores medios de operación. Los aditivos ajustados son: espato, cal, sínter, dolomía cruda, dolomía calcinada y chatarra, obteniéndose 
únicamente buenos ajustes para la cal, chatarra y dolomía cruda. Utilizando los datos procedentes de acería se han obtenido los siguientes ajustes:

\subsubsection{Dolomía calcinada}

DolomíaC $=-\mathrm{C}_{0} * \mathrm{~A}_{\text {DolomíaC }} / \mathrm{C}_{0}-\mathrm{P}_{0} * \mathrm{~A}_{\text {DolomíaC }} / \mathrm{P}_{0}+$

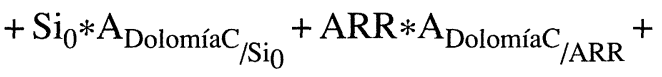

$+\mathrm{SLF} * \mathrm{~A}_{\text {DolomíaC }} / \mathrm{SLF}$

La concentración de dolomía calcinada es una función directa del contenido de silicio, aumentando cuando aumenta la cantidad de silicio y arrabio, y disminuyendo cuando aumentan los porcentajes de fósforo y carbono en el baño. La dolomía calcinada supone un aporte de $\mathrm{CaO}$ que, además, puede proporcionar un efecto refrigerante, situación que puede ser necesaria cuando aumenta la concentración de silicio, cuya reacción de eliminación es fuertemente exotérmica.

En la figura 9 se representan los valores predichos frente a los observados para la dolomía calcinada, obteniéndose un coeficiente de regresión de 0,98.

\subsubsection{Chatarra}

$$
\begin{aligned}
& \text { Chatarra }=\mathrm{C}_{0} * \mathrm{~A}_{\mathrm{CHT}} / \mathrm{C}_{0}+\mathrm{P}_{0} * \mathrm{~A}_{\mathrm{CHT}_{/ \mathrm{P}_{0}}}+ \\
& +\mathrm{S}_{0} * \mathrm{~A}_{\mathrm{CHT}} / \mathrm{S}_{0}+\mathrm{T}_{\mathrm{ARR}} * \mathrm{~A}_{\mathrm{CHT}} / \mathrm{T}_{\mathrm{ARR}}+\mathrm{IM} * \mathrm{~A}_{\mathrm{CHT}} / \mathrm{IM}+
\end{aligned}
$$

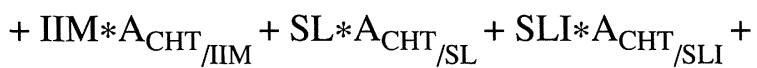

$$
\begin{aligned}
& +\mathrm{SLF} * \mathrm{~A}_{\mathrm{CHT}} / \mathrm{SLF}-\mathrm{ARR} * \mathrm{~A}_{\mathrm{CHT}} / \mathrm{ARR}
\end{aligned}
$$

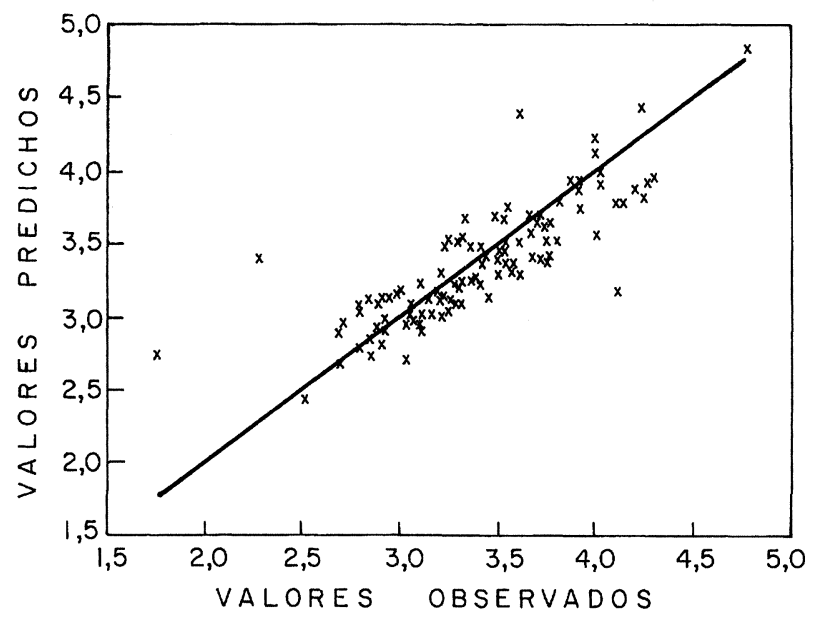

FIG. 9.- Valores predichos frente a valores observados para el ajuste de la dolomía calcinada.

Fig. 9.- Predicted values vs. observed values of the calcined dolomite.

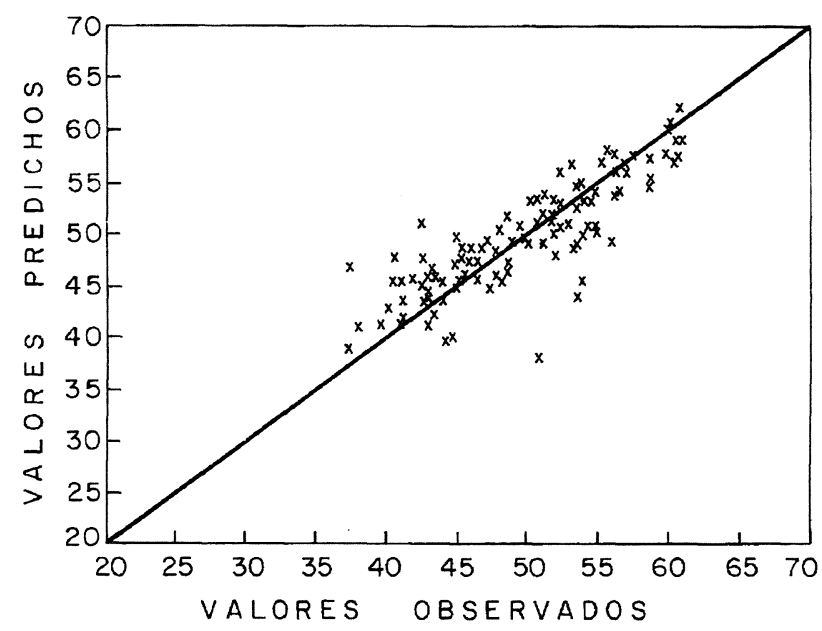

FIG. 10. - Valores predichos frente a valores observados para el ajuste de la chatarra.

Fig. 10.- Predicted values vs. observed values of the scrap.

Las variables que más influyen son la concentración inicial de carbono y la cantidad de arrabio añadida. Porcentajes elevados de carbono requieren mayor cantidad de chatarra, mientras que cantidades elevadas de arrabio necesitan menores contenidos de chatarra. La función de la chatarra es actuar como refrigerante y añadir materias primas. Por ello, cuando aumentan las concentraciones de impurezas y de oxígeno soplado, la cantidad de chatarra aumenta para actuar como refrigerante; igual tendencia se produce cuando aumenta la temperatura inicial del arrabio.

En la figura 10 se representan los valores predichos frente a los observados para la chatarra, obteniéndose un coeficiente de regresión de 0,99 .

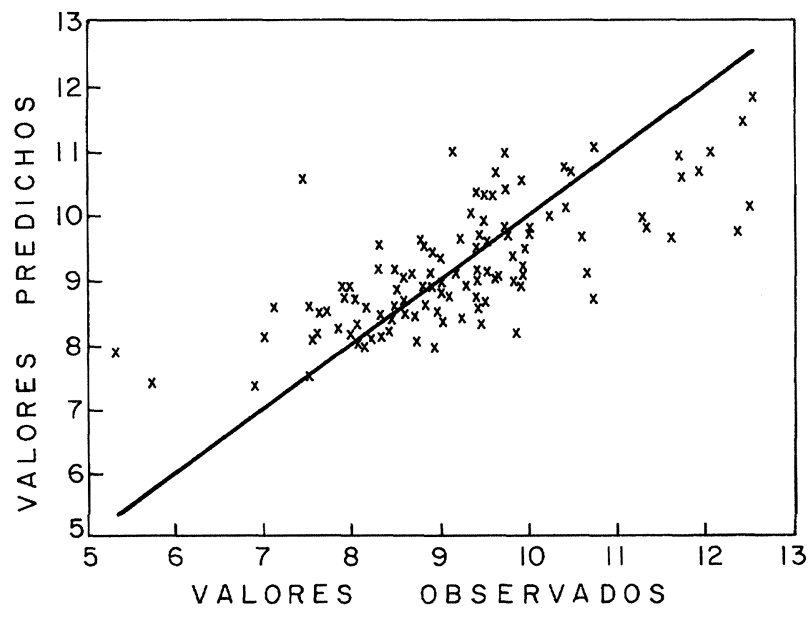

FIG. 11.- Valores predichos frente a valores observados para el ajuste de la cal.

Fig. 11.- Predicted values vs. observed values of $\mathrm{CaO}$. 


\subsubsection{Cal}

$$
\begin{aligned}
& \mathrm{Cal}=-\mathrm{C}_{0} * \mathrm{~A}_{\mathrm{Cal}_{/} \mathrm{C}_{0}}+\mathrm{Si}_{0} * \mathrm{~A}_{\mathrm{Cal}_{/ \mathrm{Si}_{0}}}+\mathrm{S}_{0} * \mathrm{~A}_{\mathrm{Cal}_{/ \mathrm{S}_{0}}}- \\
& -\mathrm{P}_{0} * \mathrm{~A}_{\mathrm{Cal} / \mathrm{P}_{0}}+\mathrm{ARR} * \mathrm{~A}_{\mathrm{Cal} / \mathrm{ARR}}-\mathrm{T}_{\mathrm{ARR}} * \mathrm{~A}_{\mathrm{Cal} / \mathrm{T}_{\mathrm{ARR}}}+ \\
& +\operatorname{ign} * \mathrm{~A}_{\mathrm{Cal}_{/ \mathrm{ign}}}+\mathrm{IIIM} * \mathrm{~A}_{\mathrm{Cal} / \mathrm{IIIM}}+\mathrm{SL} * \mathrm{~A}_{\mathrm{Cal} / \mathrm{SL}}
\end{aligned}
$$

Concentraciones elevadas de silicio, azufre y oxígeno soplado aumentan las cantidades de cal necesarias en el afino. Las variables que más influyen son los contenidos de silicio y de arrabio. Cuando aumentan la cantidad de silicio y de arrabio añadido aumenta la cantidad de cal a añadir.

En la figura 11 se representan los valores predichos frente a los observados para la cal, obteniéndose un coeficiente de regresión de 0,99 .

\section{APLICACIÓN AL CONTROL DE LOS PRODUCTOS FINALES DEL PROCESO}

En este apartado, una vez establecidos los ajustes para cada una de las variables finales del proceso, se trata de determinar los parámetros sobre los que conviene actuar para conseguir que las variables finales tomen valores comprendidos en los intervalos considerados de calidad. No todos los parámetros o variables consideradas como independientes van a poder manipularse, ya que las composiciones del arrabio procedentes del horno alto no van a poder someterse a las especificaciones deseadas. Sin embargo, los parámetros correspondientes al patrón de soplado y a los aditivos podrán variarse para conseguir los productos finales.

Como las variables dependientes se han ajustado en función de las independientes de partida, se trata de determinar sobre qué variables se puede actuar para evitar que afecten al resto de las variables dependientes que van a ser ajustadas. De acuerdo con la tabla III y observando las variables que afectan a cada una de las dependientes, se podría concluir que el control de éstas podría hacerse realizando modificaciones en las siguientes:

$$
\begin{array}{ll}
\mathrm{C}_{\mathrm{f}}= & \text { Oxígeno soplado en IM } \\
\mathrm{Mn}_{\mathrm{f}}= & \text { Oxígeno soplado en IIM } \\
\mathrm{MnO}= & \text { Oxígeno soplado en IIIM } \\
\mathrm{Fe}_{\mathrm{t}} \mathrm{O}= & \text { Sínter añadido } \\
\mathrm{CaO}= & \text { Cal añadida }
\end{array}
$$

\section{CONCLUSIONES}

El proceso de conversión con soplados apreciablemente constantes y tiempos finales muy uniformes, aunque con concentraciones diferentes en los distintos elementos, junto al hecho de disponer de un buen número de experimentos, plantea la posibilidad de la aplicación de métodos estadísticos para la obtención de resultados extrapolables para el conocimiento y control de la operación en condiciones semejantes. El análisis estadístico de los resultados del proceso en el convertidor (pocos puntos, 2 ó 3 en muchas operaciones) permite hacer deducciones empíricas de predicción que pueden servir para extrapolar los efectos de las modificaciones que se introduzcan. El ajuste limita el alcance de los sistemas que lo cumplan.

Las correlaciones obtenidas son muy aceptables para los elementos de la fase acero (C y $\mathrm{Mn})$ y de la fase escoria $\left(\mathrm{SiO}_{2}, \mathrm{P}_{2} \mathrm{O}_{5}, \mathrm{Fe}_{\mathrm{t}} \mathrm{O}, \mathrm{CaO}\right.$ y $\left.\mathrm{MnO}\right)$ pudiendo ser empleadas en la operación y control de convertidores, y en particular en la optimización de las cargas iniciales. El conocimiento de los elementos más interrelacionados, a través del análisis de varianza, permite disponer de criterios para saber sobre qué elementos actuar cuando se dispone de métodos de control distribuido.

Las relaciones obtenidas permiten determinar las acciones de control a realizar para obtener las calidades finales impuestas en cada operación. De acuerdo con los datos obtenidos, la concentración de carbono es una función del oxígeno soplado durante los minutos 3-5 de cada operación, afectando de forma negativa la cal añadida. El oxígeno soplado en momentos intermedios y finales de operación afecta al manganeso final, disminuyendo su concentración. Concentraciones elevadas de silicio dan lugar a concentraciones finales elevadas de fósforo. La concentración final de silicio es una función de la inicial y de la temperatura del arrabio, aumentando estas variables la concentración final del silicio en el arrabio.

\section{Agradecimiento}

Los autores expresan su agradecimiento a la Empresa Nacional Siderúrgica, ENSIDESA, por la información suministrada de los procesos tipo L.B.E., así como de los datos necesarios para la realización de este trabajo.

\section{SIMBOLOS}

$\begin{array}{lll}\mathrm{A}_{\mathrm{ij}} & = & \begin{array}{l}\text { Coeficientes. } \\ \text { Coefficients. }\end{array} \\ \mathrm{ARR} & = & \begin{array}{l}\text { Arrabio, t. } \\ \text { Hot metal, } t .\end{array} \\ \mathrm{CHT} & =\begin{array}{l}\text { Chatarra, } \mathrm{t} . \\ \text { Scrap, } t .\end{array}\end{array}$



DolomíaC $=$ Dolomía calcina, $\mathrm{t}$.
Dolomite burned lime, $t$.
DolomíaCr $=$ Dolomía cruda, $\mathrm{t}$.
Dolomite crude lime, $t$.
$\mathrm{Fe}_{\mathrm{t}} \mathrm{O}=$ Óxido de hierro total en la escoria $\left(\mathrm{FeO}, \mathrm{Fe}_{2} \mathrm{O}_{3}\right), \%$.
Iron oxide total in the slag ( $\mathrm{FeO}, \mathrm{Fe}_{2} \mathrm{O}_{3}$ ), \%.

ign $=$ Oxígeno soplado durante los primeros instantes, $\mathrm{Nm}^{3}$.

Oxygen blown in the first moments, $\mathrm{Nm}^{3}$.

IM = Oxígeno soplado entre los minutos 1 y $2, \mathrm{Nm}^{3}$.

Oxygen blown between 1-2 minutes, $\mathrm{Nm}^{3}$.

IIM = Oxígeno soplado entre los minutos 2 y $5, \mathrm{Nm}^{3}$.

Oxygen blown between 2-5 minutes, $\mathrm{Nm}^{3}$.

IIIM = Oxígeno soplado entre los minutos 5 y $8, \mathrm{Nm}^{3}$.

Oxygen blown between 5-8 minutes, $\mathrm{Nm}^{3}$.

$\mathrm{R}^{2}=$ Coeficiente de regresión corregido.

Corrected regression coefficient.

$\mathrm{SL}=$ Oxígeno soplado entre los minutos 8 y $10, \mathrm{Nm}^{3}$.

Oxygen blown between 8-10 minutes, $\mathrm{Nm}^{3}$.

SLI = Oxígeno soplado cuando se introduce la sublanza para tomar muestras, $\mathrm{Nm}^{3}$.

Oxygen blown when the sublance is introduced into the bath to take samples, $\mathrm{Nm}^{3}$.

SLF $=$ Oxígeno soplado durante los dos últimos minutos del proceso, $\mathrm{Nm}^{3}$.

Oxygen blown in the last two minutes of the process, $\mathrm{Nm}^{3}$.

$\mathrm{T}_{\mathrm{ARR}}=$ Temperatura del arrabio, ${ }^{\circ} \mathrm{C}$. Hot metal temperature, ${ }^{\circ} \mathrm{C}$.
$\mathrm{X}=$ Concentración del elemento disuelto en la fase metal, $\%$. Concentration of element in the metal phase, \%.

(X) $=$ Concentración del elemento disuelto en la fase escoria, $\%$. Concentration of element in the slag phase, \%.

$\mathrm{X}_{\mathrm{j}} \quad=\quad$ Variable independiente. Independent variable.

$\mathrm{Y}_{\mathrm{i}} \quad=\quad$ Variable dependiente. Dependent variable.

Subíndice (Subscript)

0

$=$ Concentración inicial de cada componente en el arrabio, $\%$.

Initial concentration in the hot metal, \%.

$=$ Concentración final de cada componente en el acero, \%.

Final concentration in the steel, $\%$.

\section{REFERENCIAS}

(1) BHAGAT, R.P., RAY H.S. y GUPTA, S.K., ISIJ Int., 31 (7), 1991: 669.

(2) Pehlke, R.D., Porter, W.F., Urban, R.F. y Gaines, J.M., "BOF Steelmaking Design", Ed. Board, 3, Nueva York, 1976.

(3) PEÑA, S.D. "Estadística. Modelos y Métodos 1. Fundamentos”, Ed. Alianza, Madrid, 1988.

(4) PeÑA, S.D. "Estadística. Modelos y Métodos 2. Fundamentos", Ed. Alianza, Madrid, 1988.

(5) Kindelan, J.M. Rev. Metal. CENIM, 1 (2), 1965: 121.

(6) Turkdogan, E.T. Trans. ISIJ, 24 (8), 1984: 591.

(7) Takawa, T., Katayama, K., Hoteiya, M. e Hirayama, N. Trans. ISIJ, 27 (12), 1987: 951.

(8) Irons, G.A., GUTHRIE, I.L. Ironmaking Steelmaking, (3), 1981: 114:

(9) Suito, H. e Inoue, R. Trans. ISIJ, 24 (4), 1984: 257.

(10) Suito, H. e Inoue, R. Trans. ISIJ, 24 (4), 1984: 301.

(11) KunisAdA, K. e IwaI, H. Trans. ISIJ, 27 (5), 1987: 332.

(12) Ono, H., Masui, T. y Mori, H. Trans. ISIJ, 25, 1985: 133.

(13) Kawai, Y., NaKaO, R. y MoRi, K. Trans. ISIJ, 24, 1984:

(14) Blanco, C. y Diaz, M. ISIJ Int., 33 (7), 1993: 757.

(15) Chatterjee, A., Linfors, N.O., y Wester, J.A. Ironmaking Steelmaking, (1), 1976, 21 